\title{
Resonant production of dark photons in positron beam dump experiments
}

\author{
Enrico Nardi, ${ }^{1, *}$ Cristian D. R. Carvajal, ${ }^{2}$ Anish Ghoshal, ${ }^{1,3}$ Davide Meloni, ${ }^{3,4}$ and Mauro Raggi ${ }^{5}$ \\ ${ }^{1}$ INFN, Laboratori Nazionali di Frascati, C.P. 13, I-00044 Frascati, Italy \\ ${ }^{2}$ Universidad de Antioquia, Instituto de Física, Calle 70 No. 52-21, Medellín, Colombia \\ ${ }^{3}$ Dipartimento di Matematica e Fisica, Università di Roma Tre, I-00146 Rome, Italy \\ ${ }^{4}$ INFN, Sezione di Roma Tre, I-00146 Rome, Italy \\ ${ }^{5}$ Dipartimento di Fisica, Università di Roma La Sapienza and INFN, Sezione di Roma, I-00185 Rome, Italy
}

(Received 2 March 2018; published 2 May 2018)

\begin{abstract}
Positrons beam dump experiments have unique features to search for very narrow resonances coupled superweakly to $e^{+} e^{-}$pairs. Due to the continued loss of energy from soft photon bremsstrahlung, in the first few radiation lengths of the dump a positron beam can continuously scan for resonant production of new resonances via $e^{+}$annihilation off an atomic $e^{-}$in the target. In the case of a dark photon $A^{\prime}$ kinetically mixed with the photon, this production mode is of first order in the electromagnetic coupling $\alpha$, and thus parametrically enhanced with respect to the $O\left(\alpha^{2}\right) e^{+} e^{-} \rightarrow \gamma A^{\prime}$ production mode and to the $O\left(\alpha^{3}\right) A^{\prime}$ bremsstrahlung in $e^{-}$-nucleon scattering so far considered. If the lifetime is sufficiently long to allow the $A^{\prime}$ to exit the dump, $A^{\prime} \rightarrow e^{+} e^{-}$decays could be easily detected and distinguished from backgrounds. We explore the foreseeable sensitivity of the Frascati PADME experiment in searching with this technique for the $17 \mathrm{MeV}$ dark photon invoked to explain the ${ }^{8} \mathrm{Be}$ anomaly in nuclear transitions.
\end{abstract}

DOI: 10.1103/PhysRevD.97.095004

\section{INTRODUCTION}

Some unquestionable experimental facts, like dark matter (DM), neutrino masses, and the baryon asymmetry of the Universe, cannot be accounted for within the standard model (SM) of particle physics. Physics beyond the SM (BSM) is thus required, which might correspond to a whole new sector containing new particles as well as new interactions. If such a sector exists, there are two possible reasons why it has not been discovered yet: (i) the mass scale of the new particles, including the mediators of the new forces, is well above the energy scale reached so far in laboratory experiments; (ii) the mass scale is within experimental reach, but the couplings between the new particles and the SM are so feeble that the whole new sector has so far remained hidden.

The first possibility keeps being actively investigated mainly in collider experiments, with the current high energy frontier set by the LHC experiments. However, the so far unsuccessful search for new heavy states has triggered in recent years an increasing interest in the second possibility, with many proposals and many new ideas to

\footnotetext{
* Corresponding author. enrico.nardi@lnf.infn.it

Published by the American Physical Society under the terms of the Creative Commons Attribution 4.0 International license. Further distribution of this work must maintain attribution to the author(s) and the published article's title, journal citation, and DOI. Funded by SCOAP .
}

hunt for new physics at the intensity frontier (see [1,2] for recent reviews). In particular, the so called dark-photon (DP) or $A^{\prime}$-boson, that is a massive gauge boson arising from a new $U(1)^{\prime}$ symmetry, can be considered as a natural candidate for a superweakly coupled new state, since its dominant interaction with the SM sector might arise solely from a mixed kinetic term $(\epsilon / 2) F_{\mu \nu}^{\prime} F^{\mu \nu}$ coupling the $U(1)^{\prime}$ and QED field strength tensors, with values of $\epsilon$ naturally falling in a range well below $10^{-2}$.

From the phenomenological point of view, light weakly coupled new particles have been invoked to account for discrepancies between SM predictions and experimental results, as for example the measured value of the muon anomalous magnetic moment [3], the value of the proton charge radius as measured in muonic atoms [4-7], or the anomaly observed in excited ${ }^{8} \mathrm{Be}$ nuclear decays by the Atomki collaboration [8-10]. This last anomaly is particularly relevant for the present paper since the new experimental technique that we are going to describe appears remarkably well suited to test, at least in some region of the parameter space, the particle physics explanation involving a new gauge boson with mass $m_{A^{\prime}} \sim 17 \mathrm{MeV}$ kinetically mixed with the photon [11].

The anomaly consists in the observation of a bump in the opening angle and invariant mass distributions of electronpositron pairs produced in the decays of an excited ${ }^{8} \mathrm{Be}$ nucleus [8], which seems unaccountable by known physics. The anomaly has a high statistical significance of $6.8 \sigma$ which excludes the possibility that it arises as a statistical fluctuation. The shape of the excess is remarkably consistent with 
that expected if a new particle with mass $m_{A^{\prime}}=17.0 \pm$ 0.2 (stat) \pm 0.5 (sys) $\mathrm{MeV}$ [10] is being produced in these decays. The strength of the $A^{\prime}$ coupling to $e^{+} e^{-}$pairs, parametrized as $\epsilon=\sqrt{\alpha^{\prime} / \alpha}$ with $\alpha^{\prime}$ the $U(1)^{\prime}$ fine structure constant, is constrained by different experimental considerations. In the Atomki setup, $A^{\prime} \rightarrow e^{+} e^{-}$decays must occur in the few $\mathrm{cm}$ distance between the target, where the ${ }^{8} \mathrm{Be}$ excited state is formed, and the detectors. This implies a lower limit $\epsilon / \sqrt{\operatorname{Br}\left(A^{\prime} \rightarrow e^{+} e^{-}\right)} \gtrsim 1.3 \times 10^{-5}$ (we will always quote limits on $\epsilon$ leaving understood that they apply to its absolute value). In the following we will assume for simplicity $\operatorname{Br}\left(A^{\prime} \rightarrow e^{+} e^{-}\right)=1$, if the $A^{\prime}$ decay with a nonnegligible rate into invisible dark particles $\chi$, with $m_{\chi}<m_{A^{\prime}} / 2$, the quoted limits need to be accordingly rescaled. However, in case the invisible decay channel becomes largely dominant, other limits different from the ones discussed in this paper apply. We refer to Ref. [12] for details.

Lower limits on $\epsilon$ much stronger than what implied by the Atomki experimental setup are obtained from electron beam dump experiments. Old data from KEK [13] and ORSAY [14] have been reanalyzed in Ref. [15] yielding, in the interesting mass range $m_{A^{\prime}} \sim 17 \mathrm{MeV}, \epsilon \gtrsim 7 \times 10^{-5}$. A stronger limit, $\epsilon \gtrsim 2 \times 10^{-4}$ was obtained in [16] from a reanalysis the E141 experiment at SLAC [17]. However, for a $m_{A^{\prime}} \sim 17 \mathrm{MeV}$ the excluded region is very close to the kinematic limit of the sensitivity (see Fig. 4) and it has been recently pointed out, by direct comparison with exact calculations [18], that the Weizsäker-Williams (WW) approximation [19-21] adopted to derive the limits become inaccurate in this kinematic region, tending to overestimate the reach in mass $[18,22,23]$. More in detail, for primary energies in the range $10-20 \mathrm{GeV}$, as was the case for the E141 beam [17], and for $m_{A^{\prime}} \sim 20 \mathrm{MeV}$, the WW approximation yields an $A^{\prime}$ production cross section about 50\% larger than the exact calculation (see Fig. 2 in Ref. [23]) and it also overestimates the $A^{\prime}$ emission spectrum at large energies (see Fig. 4 in the same reference), in which case the number of expected positrons falling within the $1.1 \mathrm{mrad}$ angular acceptance of the experiment would be overestimated both because of the larger boost, and also because of the larger lifetime dilation that would cause the $A^{\prime}$ to decay closer to the detector. Besides this, let us note that an $A^{\prime}$ slightly heavier than the benchmark value of $17 \mathrm{MeV}$ would in any case evade the E141 limit. It is then questionable if, for $m_{A^{\prime}} \gtrsim 17 \mathrm{MeV}$, the E141 constraints on the $A^{\prime}$ couplings can be considered as firmly established. Conservatively, we will assume that the corresponding region is still viable.

Upper bounds on $\epsilon$ in the relevant $A^{\prime}$ mass range also exist, see Fig. 4. The KLOE-2 experiment has searched for $e^{+} e^{-} \rightarrow \gamma A^{\prime}$ followed by $A^{\prime} \rightarrow e^{+} e^{-}$setting the limit $\epsilon<$ $2 \times 10^{-3}$ [24], while constrains from the anomalous magnetic moment of the electron [25] yield $\epsilon<1.4 \times 10^{-3}$ $[26,27]$. A comparable limit stems from $B A B A R$ searches for $A^{\prime} \rightarrow e^{+} e^{-}$decays, but it only applies for $m_{A^{\prime}}>$ $20 \mathrm{MeV}$ [28]. In summary, we will take the interval

$$
7 \times 10^{-5} \leq \epsilon \leq 1.4 \times 10^{-3} .
$$

as the window allowed for a $17 \mathrm{MeV} A^{\prime}$ decaying dominantly into $e^{+} e^{-}$. This corresponds to a DP width $2.0 \times 10^{-4} \leq \Gamma_{A^{\prime}} / \mathrm{eV} \leq 8.1 \times 10^{-2}$.

\section{THE PADME EXPERIMENT AT LNF}

Collider searches for dark photons have been carried out in electron beam dump experiments (see [15] for a review) assuming $A^{\prime}$-strahlung as the leading production mechanism in electron-nucleon scattering. Parametrically, this process is of order $\alpha^{3}$, see Fig. 1(a). As regards $A^{\prime}$ searches with positron beams, there are only few facilities which, in the next future, will be able to provide beams suitable for fixed target experiments, and correspondingly only a few experimental proposals have been put forth [29-31]. The production mechanism considered so far is analogous to the usual QED process of positron annihilation off an atomic target electron with two final state photons, where one photon is replaced by one $A^{\prime}$ see Fig. 1(b), corresponding to a process of $O\left(\alpha^{2}\right)$. This is the specific production process envisaged for the Frascati PADME experiment [31] that we will now describe briefly.

The PADME experiment [31,32] at the DAФNE LINAC Beam Test Facility (BTF) [33] of the INFN Laboratori Nazionali di Frascati (LNF) has been designed to search for DP by using a positron beam [34] impinging on a thin target of low atomic number. The $A^{\prime}$ can be detected in the

(a)

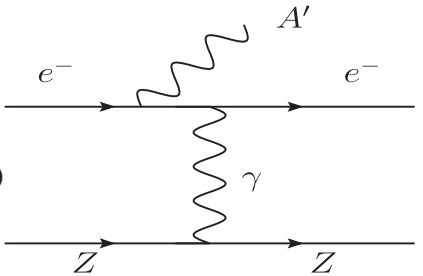

(b)

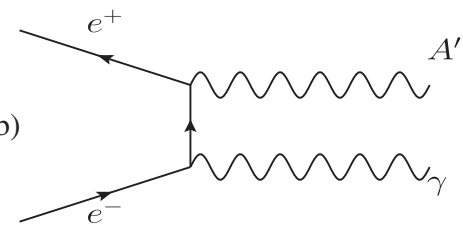

(c)

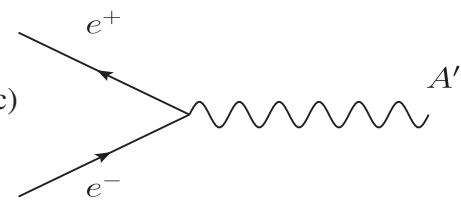

FIG. 1. $A^{\prime}$ production modes in fixed target electron/positron beam experiments: (a) $A^{\prime}$-strahlung in $e^{-}$-nucleon scattering; (b) $A^{\prime}$-strahlung in $e^{+} e^{-}$annihilation; (c) resonant $A^{\prime}$ production in $e^{+} e^{-}$annihilation. 
TABLE I. Beam parameters for the Frascati BTF.

\begin{tabular}{cccc}
\hline \hline & pot $/ \mathrm{yr}$ & $E_{\min }(\mathrm{MeV})$ & $E_{\max }(\mathrm{MeV})$ \\
\hline$e^{+}$ & $10^{18}$ & 250 & 550 \\
$e^{-}$ & $10^{18}$ & 250 & 800 \\
\hline \hline
\end{tabular}

invisible channel by searching for a narrow bump in the spectrum of the missing mass measured in single photon final states, originated via $e^{+} e^{-} \rightarrow \gamma A^{\prime}$. The experiment will use a $550 \mathrm{MeV}$ positron beam impinging on a $100 \mu \mathrm{m}$ thick active target made of polycrystalline diamond $(Z=6)$. To keep under control the counting rates the beam intensity will be kept at $\sim 10^{13}$ positrons on target per year (pot/yr), that is well below the maximum available intensity (cf. Table I). The low $Z$ and very thin target are intended to minimize the probability of photon interaction inside the target since, in order to reconstruct accurately the missing mass, the measurement requires a precise determination of the four-momentum of the $\gamma$ produced in the annihilation. The recoil photons will be detected by a quasi cylindrical calorimeter made of inorganic crystals located $3.3 \mathrm{~m}$ downstream the target, while the noninteracted positrons, which constitute the vast majority of the incoming particles, are deflected outside the acceptance of the calorimeter by a $1 \mathrm{~m}$ long dipole magnet. Three different sets of plastic scintillator bars will serve to detect electrons and positrons. Profiting by the presence of a strong magnetic field, these detectors, intended to provide an efficient veto for the positron bremsstrahlung background, can also be used to measure the charged particles momentum. The PADME detector is thus able to detect photons and charged particles and it will be sensitive to invisible $\left(A^{\prime} \rightarrow \chi \bar{\chi}\right)$ as well as to visible $\left(A^{\prime} \rightarrow e^{+} e^{-}\right)$DP decays. PADME will start taking data already during May 2018.

\section{III. $A^{\prime}$ PRODUCTION VIA RESONANT $e^{+} e^{-}$ ANNIHILATION}

In this paper we point out that for $A^{\prime}$ masses $\gtrsim 1 \mathrm{MeV}$, the process of resonant $e^{+} e^{-}$annihilation into on-shell $A^{\prime}$ depicted in Fig. 1(c), represents another production mechanism which, being of $O(\alpha)$, is parametrically enhanced with respect to the previous two production channels. Besides this, $A^{\prime}$ production via resonant $e^{+} e^{-}$annihilation has several other advantages that we will illustrate below, which altogether suggest that it might be particularly convenient to operate the PADME (as well as other) positron beam fixed target experiment in a dedicated mode in order to search for $A^{\prime}$ via resonant production. Besides experiments with positron beams, resonant $e^{+} e^{-} \rightarrow A^{\prime}$ annihilation must also be accounted for in a correct analysis of electron beam dump experiments since, as is remarked in [35], positrons are abundantly produced in the electromagnetic (EM) showers inside the dump. This feature was recently exploited in [35] in reanalysing old results from the
SLAC E137 experiment [36] by including $A^{\prime}$ production via resonant annihilation (and, but less importantly, also $A^{\prime}$ strahlung in annihilation). As a result, it was found that due to the contribution of resonant $A^{\prime}$ production, the E137 data exclude a parameter space region larger than it was previously though $[15,16]$. The extended excluded region corresponds to the area in light grey color towards the bottom of the plot in Fig. 4. Hence, in analysing electron beam dump data, $A^{\prime}$ production from annihilation of secondary positrons via the diagrams in Fig. 1(b) and (c) should be also accounted for.

In this section we consider the sensitivity of the PADME experiment to the production process $e^{+} e^{-} \rightarrow A^{\prime} \rightarrow e^{+} e^{-}$. In order to exploit the resonant production mechanism, however, an experimental setup slightly different from the one originally conceived is more convenient. The thin diamond target should be replaced by a tungsten target of several cms of length, and this for two main reasons. The first one is that of absorbing most of the incoming positron beam and of the related EM showers, and in any case to degrade sufficiently the energy of the residual emerging particles, so that the charged particles background can be easily deflected and disposed of. The $A^{\prime}$ produced in $e^{+} e^{-}$ annihilation, if sufficiently long lived, will escape the dump without interacting, and will decay inside the downstream vacuum vessel, producing an $e^{+} e^{-}$pair of well defined energy. The thick tungsten target thus allows to take advantage of the full beam intensity of $10^{18} \mathrm{pot} / \mathrm{yr}$, with a gain of five orders of magnitude with respect to the thin target running mode, see Table I. ${ }^{1}$ The second reason for using a thick target is that of providing an almost continuous energy loss for the incoming positrons propagating through the dump, so that they can efficiently "scan" in energy for locating very narrow resonances.

The energy distribution of positrons inside the BTF beam, tunable to a nominal energy $E_{b}$ within the range $250 \leq E_{b} / \mathrm{MeV} \leq 550$, can be described by a Gaussian $\mathcal{G}(E)=\mathcal{G}\left(E ; E_{b}, \sigma_{b}\right)$ where $\sigma_{b} / E_{b} \sim 1 \%$ is the energy spread. The probability that a positron with initial energy $E$ will have an energy $E_{e}$ after traversing $t=\rho \cdot z / X_{0}$ radiation lengths (with $\rho$ the density of the material in $\mathrm{g} / \mathrm{cm}^{-3}$ and $X_{0}=6.76 \mathrm{~g} / \mathrm{cm}^{-2}$ the unit radiation length in tungsten), is given by $[37,38]$

$$
I\left(E, E_{e}, t\right)=\frac{\theta\left(E-E_{e}\right)}{E \Gamma(b t)}\left[\log \frac{E}{E_{e}}\right]^{b t-1}
$$

where $b=4 / 3$ and $\Gamma$ is the gamma function. Equation (2) neglects secondary positrons from EM showers, as well as

\footnotetext{
${ }^{1}$ The maximum number of $e^{ \pm}$deliverable in one year given in the table (the one we will use) is LNF site authorization limited by the efficiency of the existing radiation shielding. However, technically the BTF could deliver up to $10^{20}$ electrons or positrons on target per year.
} 
the loss of primary positrons from $e^{+} e^{-} \rightarrow \gamma \gamma$ annihilation, but is still sufficiently accurate for our purposes. The $e^{+}$ energy distribution after $t$ radiation length is given by:

$$
\mathcal{T}\left(E_{e}, t\right)=\int_{0}^{\infty} \mathcal{G}(E) I\left(E, E_{e}, t\right) d E .
$$

Integrating $\mathcal{T}\left(E_{e}, t\right)$ in $t$ one would obtain the track-length distribution for primary positrons. However, for an accurate determination of the detectable number of $A^{\prime}$, the coordinate $z=t X_{0} / \rho$ of the production point is important, especially for the larger $\epsilon$, and hence shorter decay lengths. Thus, the integration in $t$ should be performed only when accounting for the probability of $A^{\prime}$ decaying outside the dump. We fix the origin of the longitudinal coordinate at the beginning of the dump, $z_{D}$ is the end point of the dump, and $z_{\text {det }}$ is the distance between the origin and the detector. The $A^{\prime}$ decay length $\ell_{\epsilon}=c \gamma \tau_{A^{\prime}}$, with $\gamma=\frac{m_{A^{\prime}}}{2 m_{e}}$ the time dilation factor, depends quadratically on $\epsilon$ through the lifetime $\tau_{A^{\prime}}=1 / \Gamma_{A^{\prime}}$ (but it does not depend on $m_{A^{\prime}}$, see below). For the range of $\epsilon$ given in Eq. (1), $16 \gtrsim \ell_{\epsilon} / \mathrm{mm} \gtrsim 0.04$. The number of detectable DP events then is

$N_{A^{\prime}}=\frac{N_{e^{+}} N_{0} X_{0} Z}{A} e^{-\frac{z_{D}}{\ell_{e}}} \int_{0}^{T} d t e^{\frac{X_{0}}{\rho \theta_{e}} t} \int_{0}^{\infty} d E_{e} \mathcal{T}\left(E_{e}, t\right) \sigma_{\text {res }}\left(E_{e}\right)$,

with $N_{e^{+}}$the number of incident positrons, $N_{0}$ the Avogadro number, $A=184$ the atomic mass of tungsten, $Z=74$ is the atomic number and $\sigma_{\text {res }}\left(E_{e}\right)$ the differential resonant cross section. Equation (4) takes into account the fact that the probability to detect an $A^{\prime}$ produced at $z$ is given by the integral of $d \mathcal{P} / d z=\left(1 / \ell_{\epsilon}\right) e^{-z / \ell_{\epsilon}}$ between $z_{D}-z$ and $z_{\text {det }} \rightarrow \infty$, where the limit is justified since $z_{D} \sim O(1 \mathrm{~m})$. Moreover, if the initial beam energy happens to be not much above the resonance, after just a fraction of a radiation length $\left(\rho X_{0}=3.5 \mathrm{~mm}\right.$ for tungsten) the energy of most positrons will have already degraded below the threshold for resonant production, so that setting $T=1$ for the upper limit of the integration is also a good approximation. In Eq. (4) the first exponential accounts for the fact that the larger is the length of the dump, the smallest is the number of $A^{\prime}$ that can be detected. For $z_{D} \sim 10 \mathrm{~cm}$ we can expect that virtually all the background from the EM showers will be absorbed in the dump. However, only a few $A^{\prime}$ will decay outside. To increase the statistics we can reduce $z_{D}$, but then keeping the background under control can become an issue. In the lack of a dedicated simulation of the detector/background for the resonant annihilation process, we will estimate the sensitivity to the $A^{\prime}$ couplings that could be achieved with $z_{D}=10 \mathrm{~cm}, z_{D}=5 \mathrm{~cm}$, and $z_{D}=2 \mathrm{~cm}$ (in the last two cases a reduction of the beam intensity to keep under control background contamination might be required). As regards $\sigma_{\text {res }}$, the resonant $s$-channel amplitude for $e^{+} e^{-} \rightarrow$ $A^{\prime} \rightarrow e^{+} e^{-}$does not interfere with the analogous QED process with an off-shell $\gamma$, nor with $t$-channel amplitudes that can then be neglected. Using the narrow width approximation $\sigma_{\text {res }}$ can be written as:

$$
\sigma_{\text {res }}\left(E_{e}\right)=\sigma_{\text {peak }} \frac{\Gamma_{A^{\prime}}^{2} / 4}{\left(\sqrt{s}-m_{A^{\prime}}\right)^{2}+\Gamma_{A^{\prime}}^{2} / 4},
$$

with $s \simeq 2 m_{e} E_{e}, \sigma_{\text {peak }} \simeq 12 \pi / m_{A^{\prime}}^{2}$ and $\Gamma_{A^{\prime}} \simeq \epsilon^{2} \alpha m_{A^{\prime}} / 3$. In the numerical computation we take into account $m_{e}$ effects both in the cross section and in the width, and we also account for the emission of soft photons from the initial state (see e.g., [39]) up to energies $\Delta E / E_{b} \approx 1 \%$, which can radiatively enhance the resonance width, and thus the production rate. With respect to other DP production mechanisms, resonant production has some peculiarities and advantages:

(i) The peak cross section does not depend on $\epsilon$ and the dependence of the total resonant cross section is only quadratic $\left(\sim \epsilon^{2} \alpha\right)$. As regards the observable number of electron-positron pairs from $A^{\prime}$ decays, for small $\epsilon$ the suppression in production is over-compensated by the strong enhancement from the larger decay length $\sim \exp \left(-\epsilon^{2}\right)$ which increases the number of $A^{\prime}$ that decay outside the dump. For this reason, resonant DP production in thick target experiments is particularly well suited to explore the parameter space at small $\epsilon$.

(ii) At fixed value of $\epsilon$, the $A^{\prime}$ decay length $\ell_{\epsilon}=\gamma c \tau_{A^{\prime}}$ is independent of the value of the $A^{\prime}$ mass. This is because $m_{A^{\prime}}$ cancels between the boost factor $\gamma \sim m_{A^{\prime}} /\left(2 m_{e}\right)$ and the lifetime $\tau_{A^{\prime}} \propto 1 / m_{A^{\prime}}$. For all $A^{\prime}$ masses the decay length is then fixed $\ell_{\epsilon} \sim 3 /\left(2 m_{e} \alpha \epsilon^{2}\right)$. Therefore, the entire $m_{A^{\prime}}$ range within the reach of the beam energy can be probed with the same sensitivity.

(iii) Under the reasonable assumption that the background remains constant when the beam energy is varied by only a few $\mathrm{MeV}$, the background can be directly measured from the data. This is illustrated in Fig. 2: when the beam energy lies well below the resonance, the background for $e^{+} e^{-}$pairs (assumed to be absent for the case of $z_{D}=10 \mathrm{~cm}$ in the picture) can be directly measured. When the beam energy is increased, in approaching resonant production the number of $e^{+} e^{-}$pairs produced increases in a step-wise way up to a maximum, and then remains approximately constant with increasing energy, due to positron energy losses in the material, which drive their energy towards $E_{\text {res }}$. Clearly, even in the presence of a significant number $N_{B G}$ of $e^{+} e^{-}$ 


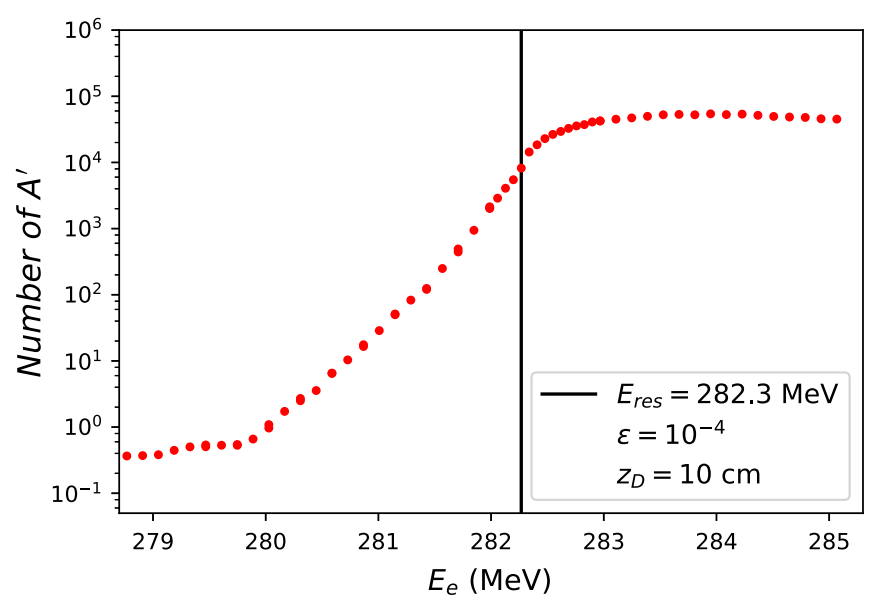

FIG. 2. The number of DP decaying outside the dump as a function of the beam energy for $\epsilon=10^{-4}$. The vertical line corresponds to the energy for resonant production of a $17 \mathrm{MeV}$ DP. A dump length $z_{D}=10 \mathrm{~cm}$ and a background free measurement have been assumed.

background pairs, as long as $N_{A^{\prime}}>\sqrt{N_{B G}}$ a signal of $A^{\prime}$ decays can be detected. ${ }^{2}$

\section{EFFECTS OF TARGET ELECTRONS VELOCITIES}

Inside materials electrons are not at rest, and in the case of large atomic numbers, like tungsten ${ }_{74} \mathrm{~W}$, electrons can have large velocities, especially the ones in the inner core shells. This can be easily verified by estimating the electrons virial velocities $\left\langle v_{n l}\right\rangle \approx \alpha Z_{\text {eff }}^{(n l)}$ in terms of the effective nuclear charge $Z_{\text {eff }}^{(n l)}$ felt by electrons in the $(n l)$ shell (a complete list of effective nuclear charges can be found in Ref. [40]). For targets of small atomic number, like ${ }_{6} \mathrm{C}$ or ${ }_{13} \mathrm{Al}$, virial velocities are small, and the effects of target electrons motion is likely to be negligible. However, for ${ }_{74} \mathrm{~W}$ one finds that the average velocities span a rather large range $0.003 \lesssim\left\langle v_{n l}\right\rangle \lesssim 0.5$ when going from valence or conduction electrons (with Fermi energy $\epsilon_{F} \sim 4.5 \mathrm{eV}$ ) to inner core electrons. Thus, for positron annihilation in tungsten the center of mass (c.m.) energy can differ sizeably from what can be naively estimated in terms of the beam energy, energy spread, energy loss due to inmatter propagation, and assuming electrons at rest. To give

\footnotetext{
${ }^{2}$ Such a spectacular signature would be prevented if the $A^{\prime}$ resonance lies somewhat below the minimum beam energy, since one would always measure $e^{+} e^{-}$resonantly produced by primary $e^{+}$degraded in energy, together with backgrounds (we thank the referee for this remark). However, in this case by raising the beam energy and stepping further away from the resonance, the number of dilepton pairs resonantly produced would drop because of the degradation of the primary beam quality due to EM showering. The behavior of a "background" which decreases with increasing beam energy would still be a signal of beyond the SM physics.
}

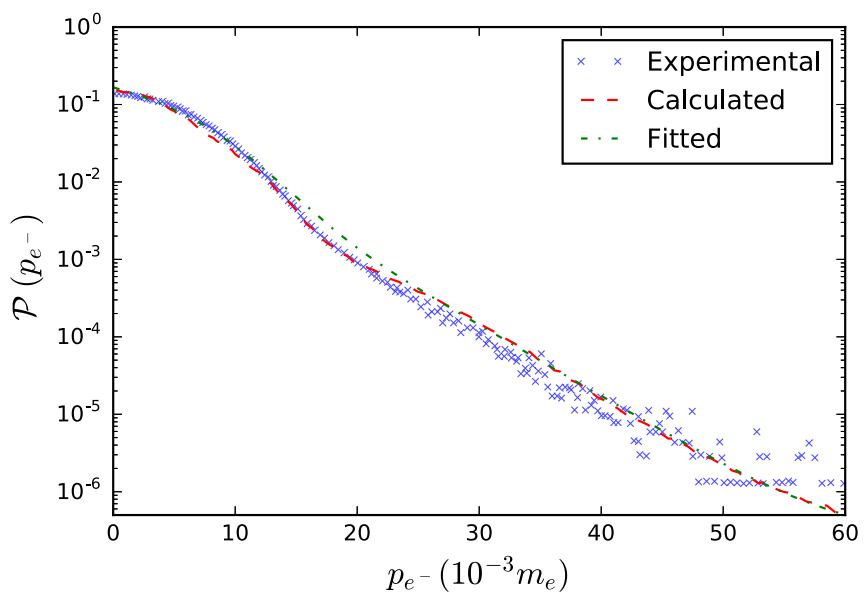

FIG. 3. The positron annihilation probability as a function of the target electron momentum for tungsten (figure adapted from Ref. [41]). The blue crosses represent experimental points, while the red dashed line is the result of the calculation method adopted in [41]. The green dot-dashed line corresponds to the fit given by the function in Eq. (6).

an example, already for a longitudinal velocity component $v_{z} \sim 0.03$ the effect of shifting the c.m. energy away from the resonant value is three time larger than the effect of the intrinsic $\sim 1 \%$ energy spread in the beam energy. Of course, what is needed to account for the c.m. energy shift is not simply the momentum distribution of electrons, but rather the positron annihilation probability as a function of the electron momentum, since annihilation with delocalized and weakly bound valence electrons, which contribute to the lowmomentum part of the momentum distribution, is more likely than annihilation with the localized and tightly bound core electrons contributing to the high-momentum part.

For positron annihilation at rest, the annihilation probability distribution as a function of the electron momentum is directly measured from the Doppler broadening by the amount $\Delta E=p_{L} / 2$ of the $511 \mathrm{keV}$ photon line, with $p_{L}$ the $e^{-}$momentum component along the direction of $\gamma$ emission (the relative direction of the two $\gamma$ 's also deviates from $180^{\circ}$ by the small angle $\left.\theta=p_{L} / m_{e}\right)$. In Fig. 3 (adapted from [41]) a large set of experimental points for ${ }_{74} \mathrm{~W}$ is represented with blue crosses. The red dashed line represents a theoretical calculation performed in the same paper. Up to $p_{e^{-}} \sim 15 \times 10^{-3} m_{e}$ the main contribution to the annihilation comes from electrons in the $5 \mathrm{~d}$ shell, beyond that point $4 \mathrm{f}$ electrons dominate, while the contribution of the high momentum core electrons becomes relevant only for $p_{e^{-}} \gtrsim 40 \times 10^{-3} m_{e}$ where, however, the annihilation probability is suppressed below $10^{-5}$. Accordingly, we find that a good fit to the experimental and calculated distributions [41] can be obtained with the sum of just three terms:

$\mathcal{P}\left(v_{e}\right)=\frac{1}{N}\left(1.015^{-v_{e}^{2}}+1.112^{-2 v_{e}}+\theta\left(v_{e}-40\right) 3 \times 10^{-6+\frac{1}{v_{e}}}\right)$, 
TABLE II. Number of $17 \mathrm{MeV}$ DP produced in the first radiation length of a tungsten target for $10^{18}$ positrons on target, for three different values of $\epsilon$. The second and third columns are for a beam energy tuned to the resonant value $E_{\text {res }}=282.3 \mathrm{MeV}$, assuming respectively electron at rest and with the velocity distribution in Eq. (6). The last column, also including $v_{e}$ effects, is for a beam energy $E_{b}=E_{\text {res }}+2 \sigma_{b}$.

\begin{tabular}{lccc}
\hline \hline$\epsilon / N_{A^{\prime}}^{\text {prod }}$ & $E_{\text {res }}\left(v_{e}=0\right)$ & $E_{\text {res }}$ & $E_{\text {res }}+2 \sigma_{b}$ \\
\hline $1.0 \times 10^{-3}$ & $7.69 \times 10^{11}$ & $1.51 \times 10^{11}$ & $4.72 \times 10^{11}$ \\
$5.0 \times 10^{-4}$ & $1.81 \times 10^{11}$ & $3.79 \times 10^{10}$ & $1.17 \times 10^{11}$ \\
$1.0 \times 10^{-4}$ & $7.25 \times 10^{9}$ & $1.49 \times 10^{9}$ & $4.73 \times 10^{9}$ \\
\hline \hline
\end{tabular}

where $v_{e}=p_{e^{-}} / m_{e}, N \sim 12$ is a normalization factor, and the first term in parenthesis accounts for $5 \mathrm{~d}$ electrons, the second for $4 \mathrm{f}$ electrons, and the last one, which is non zero only for $v_{e} \geq 40$, accounts for core electrons. To take into account target electron motion we thus replace the Mandelstam variable $s$ in $\sigma_{\text {res }}$ by

$$
s\left(v_{e}, \chi\right)=2 m_{e}\left[E_{e}\left(1-\mathcal{P}\left(v_{e}\right) v_{e} \frac{1}{2} s_{\chi} c_{\chi}\right)+m_{e}\right],
$$

where $c_{\chi}=\cos \chi$ accounts for the projection of $\vec{v}_{e}$ along the $z$-direction of the incoming positron, $s_{\chi} / 2$ with $s_{\chi}=$ $\sin \chi$ is the probability distribution for the angle $\chi$, and we integrate the cross section in $c_{\chi}$ and $v_{e} \in[0,0.06]$. Table II collects some results that illustrate how the number of DP produced within the first radiation length of tungsten depends on various effects. The second column gives the results for three different values of $\epsilon$ for a beam energy tuned at the resonant energy $E_{\text {res }}=282.3 \mathrm{MeV}$, when the motion of the target electrons is neglected. The third column gives the results obtained when the electron velocity is taken into account according to the distribution in Eq. (6). We see that the shift of the c.m. energy due to the electron momentum has the effect of reducing the number of DP produced by about a factor of five. The last column gives the results for a beam energy tuned above the resonance $E_{b}=E_{\text {res }}+2 \sigma_{b}$. The number of DP is increased by about a factor of three because of the positron energy losses, which brings on resonance also positrons in the high energy tail of the initial energy distribution.

Of course, using the annihilation probability distribution for positrons at rest in the problem at hand, is a crude way of proceeding. We can expect that target electron motion effects can be more sizeable for in-flight annihilation of short wavelength positrons with energies of $O(100 \mathrm{MeV})$, since the annihilation probability with electrons in the inner shells will be enhanced. Therefore, our estimate of the production rates might be optimistic by a factor of a few. On the other hand, while positron energy loss, which proceeds mainly via bremsstrahlung, constitute a quantized process, the dependence of the c.m. energy on the angle $\chi$ characterizing the electron momentum is continuous, and this justifies modeling positron energy losses as a continuous process.

\section{RESULTS}

Before discussing the results a few words on backgrounds are in order. The PADME spectrometers can detect $e^{+} e^{-}$pairs with good resolution for coincidence in time and momentum. The $A^{\prime}$ angular spread due to the transverse momentum of atomic electrons is much less than the intrinsic angular spread of the beam $(\sim 1 \mathrm{mrad})$ and it does not affect the reconstruction of the coincidence. For targets of sufficient thickness, background from secondary $e^{-}$ detected in coincidence with primary or secondary $e^{+}$can be avoided by measuring their depleted momentum via electromagnetic deflection. For targets of smaller length a certain number of $e^{+} e^{-}$pairs retaining a large fraction of the beam energy can exit the dump, and in this case the data driven method of searching for a "knee" in the number of $e^{+} e^{-}$pairs versus beam energy (see Fig. 2) can provide a precious tool for revealing the onset of resonant $e^{+} e^{-}$ production on top of the background. Punch-through photons, produced via bremsstrahlung in the very first layers of the dump, carrying a large fraction of the original beam energy, and converting in $e^{+} e^{-}$in the last millimeter or so, constitute the most dangerous background. This background could be significantly suppressed by equipping the experiment with a plastic scintillator veto few $\mathrm{mm}$ thick, or a silicon detector of a few hundreds of $\mu \mathrm{m}$, placed right at the end of the dump, to ensure that the $e^{+} e^{-}$pairs originate from decays in the vacuum vessel outside the dump. Additionally, if the experiment could be equipped with a suitable tracker, able to provide an accurate $e^{+} e^{-}$invariant mass reconstruction, many sources of backgrounds could be further reduced. In particular, given that the invariant mass of the $e^{+} e^{-}$originating from photon conversion $m_{e^{+} e^{-}}^{2}=0$ is very far from $m_{e^{+} e^{-}}^{2} \sim(17 \mathrm{MeV})^{2}$ expected from resonant annihilation, the punch-through photon background could be efficiently eliminated.

In Fig. 4 we show the status of the current limits for DP searches assuming visible $A^{\prime}$ decays into $e^{+} e^{-}$pairs with unit branching fraction and suppressed couplings to the proton. As is discussed in Ref. [42] the last assumption is required in order to evade the tight constraints from $\pi^{0} \rightarrow \gamma A^{\prime}$ obtained by the NA48/2 experiment [43], and to render thus viable an explanation of the ${ }^{8} \mathrm{Be}$ anomaly via an intermediate $A^{\prime}$ vector boson. For this reason we have not included in Fig. 4 the limits from the NA48/2 experiment [43] nor those from the $\nu$-Cal I experiment at the U70 accelerator at IHEP Serpukhov [44,45] which also do not apply for protophobic $A^{\prime}$. In the figure, the vertical black line gives the location of the DP resonance at $m_{A^{\prime}}=17 \mathrm{MeV}$. Leaving aside the limits from the SLAC E141 experiment for which, as explained in the introduction, the reach in $A^{\prime}$ mass might be overestimated, a 


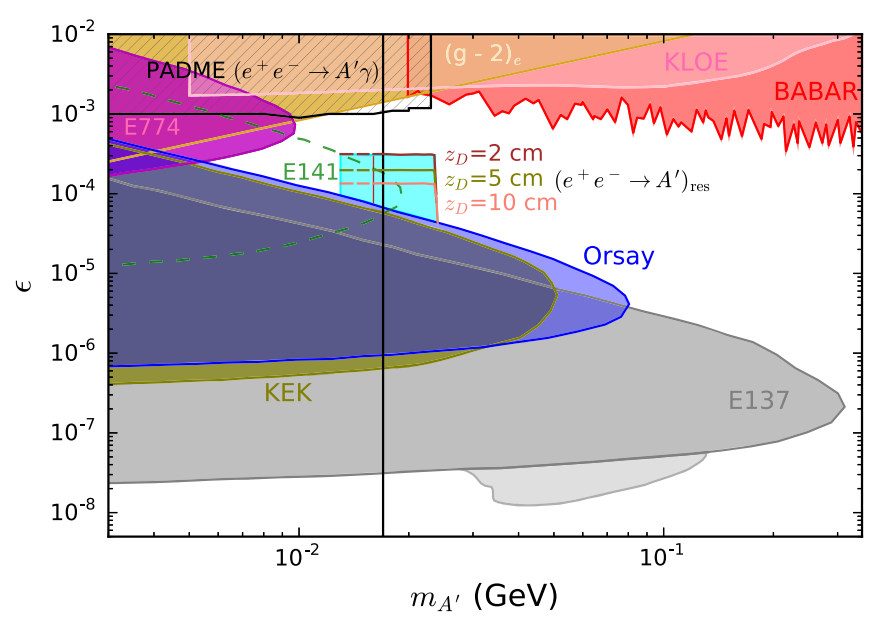

FIG. 4. Limits on the DP kinetic mixing $\epsilon$ as a function of the mass $m_{A^{\prime}}$ from different experiments. For $m_{A^{\prime}} \approx 17 \mathrm{MeV}$ (vertical black line) we consider still viable the region bounded from below by the Orsay and KEK blue and green-yellowish lines [15] and from above by the $(g-2)_{e}$ orange line [26,27]. For reasons explained in the text we do not consider as firmly excluded the region around $m_{A^{\prime}} \approx 17 \mathrm{MeV}$ delimited by the green-dashed curve of the E141 SLAC experiment $[15,16]$. The region that could be excluded by PADME running in thin target mode is hatched in black, while the three trapezoidal-shaped areas give the PADME reach in thick target mode, respectively for a 10, 5 and $2 \mathrm{~cm}$ tungsten dump, assuming zero background. These regions extend to $A^{\prime}$ masses lower than the mass corresponding to the minimum beam energy $\left(m_{A^{\prime}} \sim 16 \mathrm{MeV}\right.$ for $E_{b}^{\min }=250 \mathrm{MeV}$ depicted with the thin brown vertical line) because of positron energy losses in propagating trough the material. The lower region in light gray extending the E137 exclusion limits is from the reanalysis in Ref. [35].

viable window remains between the Orsay/KEK lines $\left(\epsilon \gtrsim 7 \times 10^{-5}\right)$ and the $(g-2)_{e}$ line $\left(\epsilon \lesssim 1.4 \times 10^{-3}\right)$. The black hatched region depicts the forecasted sensitivity of PADME in thin target mode, that will search for DP via the $e^{+} e^{-} \rightarrow A^{\prime} \gamma$ process. The limits assume $10^{13} \mathrm{pot} / \mathrm{yr}$. The light cyan trapezoidal regions represent instead the constraints that PADME could set by running in thick target mode with $10^{18} \mathrm{pot} / \mathrm{yr}$, and are respectively for tungsten targets of $10 \mathrm{~cm}, 5 \mathrm{~cm}$ and $2 \mathrm{~cm}$ of length, and neglecting backgrounds. The BTF energy range for positron beams $250 \lesssim E_{b} / \mathrm{MeV} \lesssim 550$ corresponds to c.m. energies in the interval $16 \lesssim E_{\text {c.m. }} / \mathrm{MeV} \lesssim 23.7$. Neglecting a possible small c.m. energy increase from target electron velocities, the upper value sets the upper limit on the $A^{\prime}$ masses that can be produced. The lower c.m. energy limit is indicated by the thin vertical brown line. However, because of positron energy losses, the region at low $m_{A^{\prime}}$ that can be explored extends to values smaller than $16 \mathrm{MeV}$, as indicated in the figure. Of course, in propagating well inside the dump, the beam gets degraded in energy, directions of particle momenta, number of positrons, by several effects that we are neglecting. Therefore, we can expect that the experimental sensitivity could be extended down to $m_{A^{\prime}}$ values lower than $16 \mathrm{MeV}$ by no more than a few $\mathrm{MeV}$. This might still be sufficient to reach into the region where the E141 exclusion limits can be trusted.

In summary, it is apparent how the two PADME search modes are complementary, since they can set new bounds respectively in the regions of large $O\left(10^{-3}\right)$ and small $O\left(10^{-4}\right)$ values of the DP mixing parameter $\epsilon$. With some intense and dedicated experimental efforts, the new regions in Fig. 4 could be explored in less than one year of running. In particular, the allowed window for the ${ }^{8} \mathrm{Be}$ DP could be sizeably reduced, or its existence could be unambiguously established.

\section{CONCLUSIONS}

In this paper we have suggested a new way to search for narrow resonances, and specifically $\mathrm{DP}$, coupled to $e^{+} e^{-}$ pairs, via resonant production in $e^{+} e^{-}$annihilation. There are only a few facilities around the world where positron beams in the $100 \mathrm{MeV}$ - few $\mathrm{GeV}$ range will be available. The Frascati BTF is one of those and it can provide beams with energy between $250-550 \mathrm{MeV}$. Coincidentally, this range covers precisely the c.m. energy needed to produce via resonant $e^{+} e^{-}$annihilation the $m_{A^{\prime}} \sim 17 \mathrm{MeV} \mathrm{DP}$ invoked to explain the anomaly observed in ${ }^{8} \mathrm{Be}$ nuclear transitions [8-10]. By exploiting this production process, the Frascati PADME experiment, presently under commissioning, will be able to reach well inside the interesting parameter space region. Figure 4 shows that a gap will remain between the large $\epsilon$ region that can be bounded by searching for $A^{\prime}$ produced via $e^{+} e^{-} \rightarrow \gamma A^{\prime}$, and the small $\epsilon$ region that can be efficiently explored via resonant $e^{+} e^{-} \rightarrow$ $A^{\prime}$ production. The reason for this gap is that the first process, being of $O\left(\alpha^{2} \epsilon^{2}\right)$, looses quickly sensitivity when the value of $\epsilon$ is decreased too much, while $A^{\prime}$ production via resonant annihilation becomes inefficient when $\epsilon$ becomes too large, so that most $A^{\prime} \rightarrow e^{+} e^{-}$decays occur inside the dump. Resonant $e^{+} e^{-} \rightarrow A^{\prime}$ production is not relevant for PADME running in thin target mode, because the large beam energy $E_{b} \sim 550 \mathrm{MeV}$ implies that positrons will always have energies far from any narrow resonance with mass $\lesssim 23.7 \mathrm{MeV}$, given that positron energy losses in the $100 \mu \mathrm{m}$ diamond target are negligible. However, it is conceivable that by reducing the beam energy down to $\sim 282 \mathrm{MeV}$, by increasing the size of the target to several $100 \mu \mathrm{m}$ to enhance $A^{\prime}$ resonant production, and keeping the beam intensity well below $10^{18} \mathrm{pot} / \mathrm{yr}$ to keep counting rates inside the detector under control, at least part of the remaining region for the $17 \mathrm{MeV}$ DP could be explored, and maybe the whole gap could be closed. We are presently exploring this possibility. Before concluding, we stress again that resonant $e^{+} e^{-} \rightarrow A^{\prime}$ production can be relevant also for electron beam dump experiments, since secondary positrons that could trigger the annihilation 
process are abundantly produced in EM showers. This feature has been recently exploited in reanalysing the SLAC E137 data [35], with the result of extending the previously excluded region $[15,16]$ towards smaller $\epsilon$ values, as is shown by the light gray area in Fig. 4.

\section{ACKNOWLEDGMENTS}

We thank M. Battaglieri, A. Celentano, V. Kozhuharov, L. Marsicano and P. Valente for discussions. E. N. acknowledges enlightening conversations with A. Arvanitaki, S. Dimopoulos, A. L. Morales Aramburo, M. Pospelov and
J. Pradler. The work of E. N. was supported in part by the INFN "Iniziativa Specifica" Theoretical Astroparticle Physics (TAsP-LNF), and by Perimeter Institute (PI) for Theoretical Physics. Research at P. I. is supported by the Government of Canada through the Department of Innovation, Science and Economic Development, and by the Province of Ontario through the Ministry of Research, innovation and Science. C. D. R. C. acknowledges support from COLCIENCIAS in Colombia (doctoral scholarship 727-2015), and the LNF Theory Group for hospitality and partial financial support during the development of this project.
[1] J. Alexander et al., arXiv:1608.08632.

[2] M. Battaglieri et al., arXiv:1707.04591.

[3] T. Blum, A. Denig, I. Logashenko, E. de Rafael, B. Lee Roberts, T. Teubner, and G. Venanzoni, arXiv:1311.2198.

[4] R. Pohl et al., Nature (London) 466, 213 (2010).

[5] C. E. Carlson, Prog. Part. Nucl. Phys. 82, 59 (2015).

[6] J. J. Krauth et al., arXiv:1706.00696.

[7] R. Pohl et al. (CREMA), Science 353, 669 (2016).

[8] A. J. Krasznahorkay et al., Phys. Rev. Lett. 116, 042501 (2016)

[9] A. J. Krasznahorkay et al., EPJ Web Conference 142, 01019 (2017).

[10] A. J. Krasznahorkay et al., EPJ Web Conf. 137, 08010 (2017).

[11] J. L. Feng, B. Fornal, I. Galon, S. Gardner, J. Smolinsky, T. M. P. Tait, and P. Tanedo, Phys. Rev. D 95, 035017 (2017).

[12] E. Izaguirre, G. Krnjaic, P. Schuster, and N. Toro, Phys. Rev. D 91, 094026 (2015).

[13] A. Konaka et al., Phys. Rev. Lett. 57, 659 (1986).

[14] M. Davier and H. Nguyen Ngoc, Phys. Lett. B 229, 150 (1989).

[15] S. Andreas, C. Niebuhr, and A. Ringwald, Phys. Rev. D 86, 095019 (2012).

[16] J. D. Bjorken, R. Essig, P. Schuster, and N. Toro, Phys. Rev. D 80, 075018 (2009).

[17] E. M. Riordan et al., Phys. Rev. Lett. 59, 755 (1987).

[18] Y.-S. Liu and G. A. Miller, Phys. Rev. D 96, 016004 (2017).

[19] C. F. von Weizsacker, Z. Phys. 88, 612 (1934).

[20] E. J. Williams, Kong. Dan. Vid. Sel. Mat. Fys. Med. 13, 1 (1935).

[21] K. J. Kim and Y.-S. Tsai, Phys. Rev. D 8, 3109 (1973).

[22] S. N. Gninenko, D. V. Kirpichnikov, M. M. Kirsanov, and N. V. Krasnikov, arXiv:1712.05706.

[23] D. Banerjee et al. (NA64), Phys. Rev. D 97, 072002 (2018)

[24] A. Anastasi et al., Phys. Lett. B 750, 633 (2015).

[25] M. Pospelov, Phys. Rev. D 80, 095002 (2009).
[26] M. Endo, K. Hamaguchi, and G. Mishima, Phys. Rev. D 86, 095029 (2012).

[27] H. Davoudiasl, H.-S. Lee, and W. J. Marciano, Phys. Rev. D 89, 095006 (2014).

[28] J. P. Lees et al. (BABAR), Phys. Rev. Lett. 113, 201801 (2014).

[29] I. Rachek, D. Nikolenko, and B. Wojtsekhowski, EPJ Web Conf. 142, 01025 (2017).

[30] J. Alexander, EPJ Web Conf. 142, 01001 (2017).

[31] M. Raggi, V. Kozhuharov, and P. Valente, EPJ Web Conf. 96, 01025 (2015).

[32] M. Raggi and V. Kozhuharov, Adv. High Energy Phys. 2014, 959802 (2014).

[33] A. Ghigo, G. Mazzitelli, F. Sannibale, P. Valente, and G. Vignola, Nucl. Instrum. Methods Phys. Res., Sect. A 515, 524 (2003).

[34] P. Valente, EPJ Web Conf. 142, 01028 (2017).

[35] L. Marsicano, M. Battaglieri, M. Bondí, C. D. R. Carvajal, A. Celentano, M. De Napoli, R. De Vita, E. Nardi, M. Raggi, and P. Valente, arXiv:1802.03794.

[36] J. D. Bjorken, S. Ecklund, W. R. Nelson, A. Abashian, C. Church, B. Lu, L. W. Mo, T. A. Nunamaker, and P. Rassmann, Phys. Rev. D 38, 3375 (1988).

[37] H. Bethe and W. Heitler, Proc. R. Soc. A 146, 83 (1934).

[38] Y.-S. Tsai and V. Whitis, Phys. Rev. 149, 1248 (1966).

[39] M. Bohm and W. Hollik, Nucl. Phys. B204, 45 (1982).

[40] E. Clementi, D. L. Raimondi, and W. P. Reinhardt, J. Chem. Phys. 47, 1300 (1967).

[41] V. J. Ghosh, M. Alatalo, P. Asoka-Kumar, B. Nielsen, K. G. Lynn, A. C. Kruseman, and P. E. Mijnarends, Phys. Rev. B 61, 10092 (2000).

[42] J. L. Feng, B. Fornal, I. Galon, S. Gardner, J. Smolinsky, T. M. P. Tait, and P. Tanedo, Phys. Rev. Lett. 117, 071803 (2016).

[43] J. R. Batley et al. (NA48/2), Phys. Lett. B 746, 178 (2015).

[44] J. Blümlein and J. Brunner, Phys. Lett. B 731, 320 (2014).

[45] J. Blümlein and J. Brunner, Phys. Lett. B 701, 155 (2011). 